\title{
Klinischer Fortschritt: Forschung muss beim Patienten ankommen
}

Nicht alles, was als Fortschritt reklamiert wird, kommt tatsächlich dem Patienten zugute, entweder, weil es nicht wissenschaftlich geprüft und seine Wirkung unsicher ist - wie die meisten „Disease Management Programme“ (DMP) der Krankenkassen - oder weil es aus unterschiedlichen Gründen seinen Weg in die medizinische Anwendung nicht findet, wie zum Beispiel ein in einer kontrollierten Studie geprüftes DMP für die Herzinsuffizienz. Hier kann nicht mit Kosten argumentiert werden, sondern es geht um den Stellenwert der Wissenschaftlichkeit in der Versorgung. Fortschritt in der Medizin bedarf zum einen der klinischen Forschung und zum anderen der Überprüfung, inwieweit Studienergebnisse sich im Alltag reproduzieren lassen, also der Versorgungsforschung. Mit den Methoden der Versorgungsforschung lassen sich über die Wirkung einzelner Medikamente hinaus, diagnostische oder therapeutische Konzepte überprüfen. Es lässt sich auch klären, inwieweit und wie rasch Forschungsergebnisse in der medizinischen Versorgung ankommen.

Das Programm „Klinische Studien“, gemeinsam getragen von der Deutschen Forschungsgemeinschaft (DFG) und dem Bundesministerium für Bildung und Forschung (BMBF) hat auch in Deutschland zu einem Niveau der patientenorientierten Forschung geführt, das alle Qualitätskriterien erfüllt. Das Programm ist eine Erfolgsstory und hat es geschafft, patientenorientierte klinische Forschung in Deutschland international konkurrenzfähig zu machen. Klinische Forschung und Versorgungsforschung ringen nach wie vor in Deutschland um Anerkennung und geeignete Finanzierung. Die Einrichtung von organbezogenen Forschungszentren, zum Teil geleitet von Grundlagenforschern, soll in Zukunft auch klinische Studienprogramme aufbauen oder ihre Betreuung übernehmen. Diese Entwicklungen sind gerade für eine internistische Forschung, die unterschiedliche Organdisziplinen zusammenführt und im Zeitalter einer hohen und zunehmenden Lebenserwartung und Multimorbidität eines großen Bevölkerungsanteils von besonderer Bedeutung ist, wahrscheinlich nicht förderlich. Mit Unterstützung des BMBF eingerichtete und inzwischen erprobte klinische Forschungsstrukturen wie die Interdisziplinären Zentren für Klinische Forschung (IZKF) oder die Integrierten Forschungs- und Behandlungszentren (IFB), die eine Mission für die interdisziplinäre klinische Forschung erfüllen, könn- ten mit der Beendigung eines offen ausgeschriebenen Studienprogramms die Grundlage ihrer Förderung verlieren.

Die Deutsche Gesellschaft für Innere Medizin versteht es als ihren Auftrag, wissenschaftliche Erkenntnisse in angewandte Heilkunde zu übertragen und so die Fortschritte der Inneren Medizin unmittelbar dem Patienten zugutekommen zu lassen. Diesem Grundsatz folgen auch die DGIM-Gesellschaftsausgaben der DMW, die neueste Forschungsergebnisse und ihre Bedeutung für die Praxis zusammenfassen.

Von den Schwerpunkten der Inneren Medizin lassen sich höchstens die Kardiologie, die Nephrologie, die Pneumologie und vielleicht noch die Angiologie auf ein Organ festlegen, alle anderen beschäftigen sich a priori entweder mit Organsystemen, mit Systemerkrankungen oder sind Querschnittsfächer wie die Geriatrie und die Intensivmedizin. Beiträge dieser DGIM-Gesellschaftsausgabe berichten aber, dass die kardiovaskuläre Komorbidität bei Psoriasis-Arthritis hoch, dass sie bei COPD ein wichtiger Prognosefaktor ist, dass Diabetiker bei der PTA von Drug Eluting Stents profitieren und dass die Dialyse bei Herzinsuffizienz und Leberzirrhose wirksam sein kann. Diese Zitate sollen verdeutlichen, wie wichtig eine internistische Betrachtungsweise für die Diagnostik und Therapie unserer Patienten ist. Sie weisen aber auch auf einen dringlichen Forschungsbedarf hin, denn die meisten klinischen Studien schließen multimorbide Patienten aus. Ein anderes Thema dieses Heftes, das fortgeschrittene Lungenkarzinom, weist die Palliativmedizin als wichtiges Forschungsgebiet aus. Palliativmedizin wird in der Öffentlichkeit häufig als ethisches und politisches Problem gesehen, der Mangel an überprüften Konzepten wird dabei kaum wahrgenommen.

Die Herausgeber der DGIM-Gesellschaftsausgaben der DMW sorgen für die Kommunikation neuester Ergebnisse der klinischen Forschung und ihrer Bedeutung für die Patientenversorgung. Aber auch Lücken im Kenntnisstand werden diskutiert und notwendige Projekte und Perspektiven der klinischen Forschung aufgezeigt. Heute wollten wir auf ein generelles Defizit in der interdisziplinären organübergreifenden „internistischen Forschung“ hinweisen, die durch die langfristige Einrichtung von Organforschungszentren durch das BMBF möglicherweise weiter ins Abseits gerät.

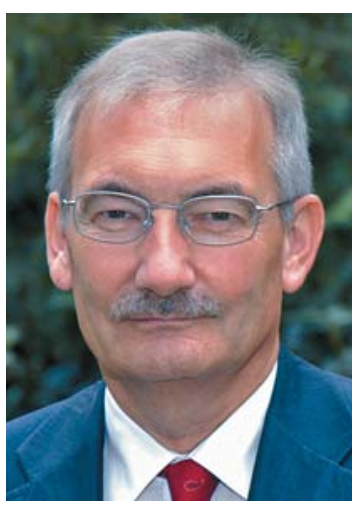

G. Ertl

Institut

Medizinischen Klinik und

Poliklinik I,

Universitätsklinikum Würzburg

Bibliografie

Dol 10.1055/s-0031-1286377 Dtsch Med Wochenschr 2011 . 136: 1993 - (C) Georg Thieme Verlag KG Stuttgart - New York . ISSN 0012-0472

Korrespondenz

Prof. Dr. Georg Ertl

Medizinische Klinik I

Klinikum der Julius-Maximilians-

Universität

Josef-Schneider-Str. 2

97080 Würzburg 